\title{
E-learning Emergency? A student-teacher dialogue on sudden shifts to online medical education
}

\author{
Yeung, A.N.M. ${ }^{1} \&$ See, C. ${ }^{2}$
}

\begin{abstract}
Background: The COVID-19 pandemic of 2020 has forced medical educators worldwide to make sudden and unexpected shifts from face-to-face teaching to e-learning. These emergency changes can be quite unlike coordinated and planned shifts to digital education, and some institutions have found themselves with limited time for preparation of teachers and students, as well as digital infrastructure. This article explores issues raised through a series of student-teacher dialogues. These were conducted online and outside of the formal curriculum development processes as a form of rapid and informal exchange on the subject of the sudden shift to e-learning. Three salient issues were raised from our iterant discussions; the first of which being the generation gap leading to expectation mismatches of students between media they normally consume and those which teachers are able to produce. We propose mutual understanding and sharing of best digital practices as methods to reduce the degree of mismatch. The second issue was the potential benefits and pitfalls of students as co-creators of elearning. Potential benefits of technical proficiency in e-learning production and a peer-teaching element must be balanced with respect for students' time and effort in any such endeavours. Finally, the issue of wealth inequality within medical student bodies was identified, which is likely to be exacerbated by lack of access to equalising provisions such as university computing facilities. This article serves to highlight issues that teachers and students face in this emergency e-learning era, and to demonstrate how informal student-teacher dialogue can identify issues for further scholarly exploration.
\end{abstract}

Keywords: E-learning, student-teacher dialogue, COVID-19, wealth inequality, student co-creators, online learning.

\section{Introduction}

Hong Kong medical schools have experienced three abrupt transitions from in-class teaching to purely online classes; during the 2003 SARS outbreak (Patil \& Yan, 2003), the 2019 AntiExtradition Law Protests and the COVID-19 pandemic in 2020 (Ahmed, Allaf \& Elghazaly, 2020). Unlike well-planned transitions to elearning featuring pilot studies and progressive implementation, we are in the grip of 'emergency e-learning' where Faculty simply has to make do with current digital facilities,

\section{${ }^{1} L K S$ Faculty of Medicine, The University of Hong Kong, Hong Kong \\ ${ }^{2}$ School of Biomedical Sciences, Faculty of Medicine, The} Chinese University of Hong Kong, Hong Kong

Corresponding Author - Dr Christopher See, Lecturer, School of Biomedical Sciences, Chinese University of Hong Kong, Hong Kong

Email: christophersee@cuhk.edu.hk their teaching experience and the medical education literature, and students have to live with the results. In our medical schools, a range of classes, lectures, practical sessions, dissections and PBL tutorials have all been shifted to online formats via streaming or recorded online videos, varying wildly in quality and the warmth of student reception.

The aim of this article is to share our experience and identify unexpected or important issues from both the student and teacher perspectives emerging from sudden transitions to medical education in a fully online form. It aims to initiate discussion, set a course for enhanced mutual understanding and highlight avenues for further exploration and scholarship in medical education. In a manner befitting the times, this article was written purely online through several iterative discussions via Zoom and collaborative writing on Google Docs. We believe the following three points are particularly salient. 


\section{Bridging the generational gap}

A key issue of the e-learning era is that students may consume a wide range of educational resources beyond Faculty-provided resources via platforms such as YouTube, Instagram and Facebook. The quality of such materials can vary vastly, as can their acceptability to students, but amongst all of them are resources which students might prefer to use. One issue emerging from our discussion is that Faculty members, despite their role as producers, may be much less familiar with materials which already exist. This train of thought results in a fundamental educational question - "Which Instagram accounts do I forward to my lecturer?' The answer, we hope, is as many as possible to broaden the horizons of Faculty members, and particularly help them to bridge generational gaps by experiencing material consumed by the students of today (Ruzycki et al., 2019). We also hope that students are empathetic to some technical and experiential aspects of producing good videos that their teachers may stumble across. YouTubers may have produced dozens or hundreds of clips with studio-quality equipment, whilst for sudden elearning changes, teachers may be sitting behind a laptop recording via webcam for the first time ever.

\section{Students as important but voluntary co- creators of e-learning}

We recognise that students often have greater expertise in digital creation than Faculty members. It is also increasingly recognised that peer-led education benefits medical students both receiving and giving such teaching ( $\mathrm{Yu}$ et al., 2011). So why don't medical schools simply involve students at every stage of co-creation? We believe there are some significant complexities when it comes to implementation. Given the amount of time it takes to prepare, film, edit and quality-control good e-learning materials, students might quite reasonably ask "What is in it for me?" There may be a broad range of student opinions on this matter, but simply compelling students to contribute would not be an acceptable system. A better way might be to offer a range of incentives for students, including awards, scholarships or grants for the best student-created materials.

A dialogue between Faculty and the student body is important in aligning the teaching goals with student contributions in a manner that respects the value of their time and efforts. What about other 'hierarchical' issues, such as authorship order arising from educational research output? It is easy for Faculty to 'spend' student time on their behalf, but we must recognise and reward the individuals involved appropriately. We take the view that learning to teach is part of the journey of becoming a doctor; however, that journey has many possible avenues and so we are keen to promote involvement but not uniformity of student co-creators.

\section{Compounding wealth inequality amongst medical students}

One of the most serious and pernicious issues arising from our dialogue was the potential for the broadening of the rich-poor divide by means of campus shutdown and enforced e-learning. It may be supposed that medical schools can provide adequate resources to circumvent wealth inequalities through comprehensive hardware and software support. However, less well-off students may suffer from e-learningspecific disadvantages such as poor internet connectivity, slower mobile devices and smaller screens in the absence of the equalising effects of university computing facilities. This may be particularly anxiety-inducing in cases of remote assessments requiring anti-cheating software.

Other wealth-associated factors such as size and privacy of study areas available at home may also play a role in broadening student inequality in the e-learning era when students no longer have access to common learning areas. The conversation on wealth inequality as a barrier to entry to medical school is gaining recognition (Baugh, Vanderbilt \& Baugh, 2019), but we contend that issues of wealth inequality once entering medical school may be overlooked. Such phenomena are poorly studied in the medical education literature and future studies of the entire student learning ecosystem including wealth-related factors could be helpful in making sure medical students have equality of opportunity throughout their learning journey.

\section{Conclusion}

This dialogue was specifically designed to bypass the normal institutional feedback systems and allow frank and informal feedback to be passed from student to teacher. We aimed to share some reflections which are not well examined in the extant medical education literature and raise some issues worthy of further investigation and research. We would particularly like to alert students and Faculty to issues around wealth inequality which may be exacerbated during campus shutdowns and e- 
learning-only studies. We also wish to underline the power of student-Faculty co-creation with the insight and enjoyment that working together can bring, alongside the potential for issues. In this new e-learning era, the fact that we are on opposite sides of the screen does not mean we are on opposing sides.

\section{References}

Ahmed, H., Allaf, M. \& Elghazaly, H. 2020. COVID-19 and medical education. The Lancet Infectious Diseases. 20(7), pp.777778.

Baugh, A.D., Vanderbilt, A.A. \& Baugh, R.F. 2019. The dynamics of poverty, educational attainment, and the children of the disadvantaged entering medical school.
Advances in Medical Education and Practice.10, pp.667-676.

Patil, N.G. \& Yan, Y.C.H. 2003. SARS and its effect on medical education in Hong Kong. Medical Education. 37(12), pp.1127-1128.

Ruzycki, S.M., Desy, J.R., Lachman, N. \& Wolanskyj-Spinner, A.P. 2019. Medical education for millennials: How anatomists are doing it right. Clinical Anatomy. 32(1), pp.20-25.

Yu T.C., Wilson, N.C., Singh, P.P., Lemanu, D.P., Hawken, S.J. \& Hill, A.G. 2011. Medical students-as-teachers: a systematic review of peer-assisted teaching during medical school. Advances in Medical Education and Practice. 2, p.157. 